\title{
Factores determinantes de la reducción de la desigualdad en la distribución de la renta en países de América Latina
}

\author{
Carmen Ramos Carvajal, Mercedes Alvargonzález Rodríguez \\ y Blanca Moreno Cuartas
}

\section{Resumen}

Este trabajo procura analizar las pautas de la desigualdad de renta y los factores determinantes de su evolución en países de América Latina durante el período 2004-2013. Inicialmente, se determina la desigualdad en el reparto del ingreso en algunos países de América Latina mediante el índice de Theil y se observa que en la mayoría de ellos la desigualdad ha disminuido en el período considerado. Asimismo, se propone un modelo econométrico de datos de panel para estudiar los factores determinantes del nivel de desigualdad. El PIB per cápita, el gasto sanitario per cápita, la presión fiscal, la tasa de pobreza, la tasa de alfabetización y los años de estudio son variables estadísticamente significativas para explicar la desigualdad. Se aplican técnicas multivariantes con el objeto de clasificar los países considerados en grupos, según el nivel de desigualdad, estableciéndose una clasificación de acuerdo con su posición para disminuir el nivel de inequidad.

\section{Palabras clave}

Distribución del ingreso, igualdad, medición, modelos econométricos, análisis de factores, América Latina

\section{Clasificación JEL}

C23, O15, 054

\section{Autoras}

Carmen Ramos Carvajal es Profesora Titular en la Facultad de Economía y Empresa del Departamento de Economía Aplicada de la Universidad de Oviedo, España. Correo electrónico: cramos@uniovi.es.

Mercedes Alvargonzález Rodríguez es Profesora Titular en la Facultad de Economía y Empresa del Departamento de Economía Aplicada de la Universidad de Oviedo, España. Correo electrónico: malvarg@uniovi.es.

Blanca Moreno Cuartas es Profesora Titular en la Facultad de Economía y Empresa del Departamento de Economía Aplicada de la Universidad de Oviedo, España. Correo electrónico: cramos@uniovi.es. 


\section{Introducción}

Una de las características económicas más documentadas de los países de América Latina es la que se refiere a los altos niveles de desigualdad en la distribución de la renta de las familias. Como señalan Gasparini y Gluzmann (2012), la mayoría de los países de esta región han estado siempre entre las sociedades más desiguales del mundo desarrollado. Los niveles de inequidad en Europa y los Estados Unidos, incluso en los períodos de crisis, han sido y continúan siendo mucho más reducidos que en América Latina, como se señala en Ayala (2013).

Se entiende por desigualdad económica la disparidad fundamental que permite a una persona ciertas oportunidades materiales y se las niega a otra. Las medidas relativas a la desigual distribución de la renta permiten conocer cómo se produce el reparto del crecimiento y el desarrollo entre los distintos grupos sociales.

La distribución de la renta constituye un elemento fundamental para determinar las dinámicas que generan el crecimiento económico y el bienestar de la población. La distribución equitativa de la renta es una de las características de las sociedades desarrolladas, mientras que las economías menos desarrolladas suelen tener un reparto más desigual de la renta y la riqueza. Este es, sin duda, un tema recurrente en la literatura, dada su relevancia y sus implicaciones en el bienestar de los individuos.

Parece lógico pensar que la desigualdad se incrementa en situaciones de crisis económica. Sin embargo, en distintos estudios se ha mostrado que el binomio desigualdad-crisis no siempre aparece y que el nivel de desigualdad está muy influenciado, además de por la situación económica, por la estructura y las características de cada territorio, lo que impide que se dé una vinculación directa generalizada entre ambos aspectos (Atkinson y Morelli, 2011; Adiego y Ayala, 2013).

Las aproximaciones al estudio conceptual de la desigualdad han sido variadas. Entre otros, se destacan los trabajos de Cowell (1977), Nygard y Sandström (1981), Foster (1983), Zubiri (1985), Ruiz-Castillo (1987), Pena y otros (1996) y Dagum (2001).

También se pueden señalar autores que han analizado, desde una perspectiva más aplicada y relativa a América Latina, la desigualdad económica y social, como por ejemplo y sin pretender ser exhaustivos: Ariza y de Oliveira (2007), Martín (2008), Azevedo y otros (2013), Lustig, López-Calva y Ortiz-Juárez (2013), Gasparini y Gluzmann (2012), y Morgan y Kelly (2013).

Sin embargo, y a pesar de los elevados niveles de inequidad en América Latina, si se analizan las estadísticas sobre desarrollo económico, se puede señalar que durante la primera década del presente siglo la región experimentó un crecimiento económico notable y, a la vez, una reducción de los niveles de desigualdad. Esto puede deberse a la aplicación de políticas de transferencias y a las inversiones en salud y educación (Perticara, 2012).

El principal objetivo de este trabajo es analizar las pautas de la desigualdad de renta y los factores determinantes de su evolución en países de América Latina durante los últimos años.

Con este propósito, en las secciones II y III se aborda el estudio de la desigualdad en el reparto de la renta en los países latinoamericanos en el período 2004-2013, mediante la utilización de una de las medidas habitualmente empleadas con este fin: el índice de Theil.

En la sección IV se lleva a cabo la descomposición de la desigualdad global en la región, considerada en dos componentes: la desigualdad existente dentro de cada país y las desigualdades que se presentan entre los distintos países. Dicha descomposición permitirá analizar cuál de estas componentes ha tenido más relevancia en la desigualdad total.

En la sección $V$ se estima el impacto de algunas variables - como el PIB, la educación, el gasto sanitario, la pobreza y la presión fiscal - sobre la reducción de la desigualdad en los países de 
América Latina durante el período señalado. Para realizar dicha estimación se utiliza la metodología de los datos de panel, que permite controlar los efectos inobservables específicos de cada país.

Para completar este trabajo, en la sección VI se aplican técnicas multivariantes, como los análisis clúster y factorial. La utilización de la primera técnica proporciona una clasificación de los países en grupos que tengan pautas de comportamiento similares respecto de las variables consideradas. El análisis factorial, por su parte, permite sintetizar la información en un indicador con el que se pueda determinar qué países estarán en mejor posición para reducir el nivel de desigualdad.

Por último, en las conclusiones que se incluyen en la sección VII se recopilan los principales resultados obtenidos en este trabajo.

\section{Desigualdad: medida y descripción de su evolución}

En la literatura sobre el tema existe un número muy amplio de indicadores de desigualdad. En este trabajo se usará el índice de Theil, ya que verifica una propiedad de gran interés en los estudios empíricos: la descomponibilidad. El índice de Gini, medida frecuentemente utilizada, presenta la limitación de no ser descomponible, es decir, si se dispone de una población dividida en grupos no es posible obtener la desigualdad global a partir de la desigualdad interna de los grupos y de la desigualdad entre grupos.

A continuación, se define el índice de Theil (1967): sea X la variable renta que toma valores $\left\{x_{1}, \ldots, x_{M}\right\}$ con frecuencias relativas $\left\{f_{1}, \ldots, f_{M}\right\}$, se denota por $\mathrm{E}(\mathrm{X})$ al valor esperado de $\mathrm{X}$, es decir, la renta per cápita de la población ${ }^{1}$. El índice de Theil (IT) viene dado por la siguiente expresión:

$$
\operatorname{IT}(X)=\frac{1}{E(X)} \sum_{i=1}^{M} x_{i}\left[\log \frac{x_{i}}{E(X)}\right] f_{i}=\frac{1}{E X)} \sum_{i=1}^{M} x_{i}\left[\log \left(x_{i}\right)-\log (E(X))\right] f_{i}
$$

Este indicador puede interpretarse como la media ponderada de las desviaciones entre el logaritmo de la renta y el logaritmo de la renta per cápita de la población.

El empleo de la función logarítmica tiene como principal ventaja permitir asignar una mayor importancia a las rentas más bajas, lo que resulta adecuado desde la concepción normativa de la desigualdad como problema social.

En el caso de equidistribución, las rentas de los individuos coincidirían con su valor esperado y el índice tomaría el valor nulo, mientras que en el caso opuesto, en el que una sola persona acumulase toda la renta, el índice estará acotado superiormente por Mlog(M).

El índice de Theil es un indicador adecuado de la desigualdad, ya que verifica un conjunto de propiedades que se consideran deseables ${ }^{2}$. Entre estas propiedades se encuentran la continuidad, la simetría y el principio de transferencias de Pigou-Dalton (esto es, si se transfiere parte de la renta de una persona rica a una pobre, sin dejar de preservar el orden de los rangos de ingresos, la desigualdad no debe aumentar) $)^{3}$. También verifica el principio del decrecimiento del impacto ante transferencias progresivas, es decir, el valor se reduce en mayor cuantía cuando las transferencias progresivas involucran a individuos cada vez más próximos a la "cola" baja de la distribución (siempre que estas sean de igual cuantía y se realicen entre individuos cuyo diferencial de rentas es idéntico). Además,

\footnotetext{
1 Theil fue el primer autor que propuso las medidas de información estadística como marco adecuado para el estudio de la desigualdad, basándose en razones de tipo conceptual y operativo.

2 Shorrocks (1980) fue uno de los autores que estudió las propiedades del índice de Theil.

3 En la forma más fuerte de esta propiedad, el nivel de la desigualdad debe disminuir.
} 
cumple la no homoteticidad distributiva, esto es, dada una renta total constante, a medida que la desigualdad aumenta, se concede mayor importancia a la situación de los individuos más pobres.

Por último, este índice verifica, como ya se ha señalado, la propiedad denominada "descomponibilidad". Su cumplimiento exige que exista una relación coherente entre el nivel de desigualdad total de la economía y el de los subgrupos que la componen. Si se dispone de la información para M países, el índice de Theil viene dado por la expresión:

$$
I T(X)=\sum_{P=1}^{M} I T_{P}(X) \frac{E_{P}(X)}{E(X)} \frac{N_{P}}{N}+\sum_{P=1}^{M} \frac{E_{P}(X)}{E(X)} \frac{N_{P}}{N} \log \left(\frac{E_{P}(X)}{E(X)}\right)
$$

donde $I T_{P}(X)$ es el índice de Theil del país $P, N_{P}$ es la población del país $P, N$ es la población global, $E(X)$ es la renta per cápita global y $E_{P}(X)$ es la renta per cápita del país $P$.

El primer sumando de la expresión (2) recoge la desigualdad interna de los países y el segundo la discrepancia entre los distintos países.

\section{La desigualdad de la renta en los países de América Latina}

América Latina comprende países del continente americano donde prevalecen los idiomas de raíz latina, como el español y el portugués, esto es, México, casi toda Centroamérica (Costa Rica, El Salvador, Guatemala, Honduras, Nicaragua y Panamá) y casi todas las naciones de América del Sur (Argentina, Bolivia (Estado Plurinacional de), Brasil, Chile, Colombia, Ecuador, Paraguay, Perú, Uruguay y Venezuela (República Bolivariana de)). El resto de los países continentales de Centroamérica y América del Sur (Belice, Guyana, Suriname y el territorio de la Guayana Francesa) en general no se consideran parte de América Latina, pues pertenecen, por vínculos culturales y económicos, a la región del Caribe. Existen países americanos de colonización latina ubicados en el Mar Caribe: Cuba, Puerto Rico y la República Dominicana, de origen hispano, y Haití, de colonización francesa.

En este artículo se trabaja con 15 países de América Latina continental de los señalados anteriormente, más la República Dominicana. Se excluye a Cuba, Honduras, Nicaragua y Venezuela (República Bolivariana de), por no disponer de la información necesaria. Puerto Rico tampoco es considerado, ya que se trata de un territorio asociado a los Estados Unidos, y Haití no se incluye por tratarse de un país más ligado culturalmente al Caribe que a América Latina y por tener un sistema de encuestas de hogares muy precario. En resumen, los países considerados en este artículo son: Argentina, Bolivia (Estado Plurinacional de), Brasil, Chile, Colombia, Costa Rica, Ecuador, El Salvador, Guatemala, México, Panamá, Paraguay, Perú, República Dominicana y Uruguay.

\section{Reconstrucción de una base de datos sobre desigualdad: primeros resultados}

Para cuantificar la desigualdad de renta en América Latina se utiliza el índice de Theil, ya que cumple las propiedades anteriormente señaladas y se ha empleado en un buen número de estudios (Villaverde, 1996; Duro, 2004; Goerlich y Mas, 2004; Martín, 2008; Azevedo y otros, 2013, y Amarante, Galván y Mancero, 2016, entre otros).

El período en el que se lleva a cabo este estudio es de diez años (de 2004 a 2013) y se considera lo suficientemente amplio como para realizar un análisis riguroso sobre la desigualdad de la renta. 
Se comenzó por recabar la información sobre la desigualdad de los países señalados en el período de tiempo de estudio, para lo cual se consideraron datos relativos al índice de Theil proporcionados por el Banco Mundial y la Base de Datos Socioeconómicos para América Latina y el Caribe (SEDLAC).

Desafortunadamente, la información obtenida de estas fuentes estadísticas no es completa y se han debido estimar los datos no disponibles para algunos países en ciertos años. Gómez, Palarea y Martín (2006) establecen una clasificación de diferentes técnicas de imputación de datos, como la consideración de la media, la regresión, la regresión estocástica y otros métodos más complejos basados en las verosimilitudes ${ }^{4}$. Estos últimos, aunque ofrecen la mejor alternativa, "en determinadas situaciones, (...) pueden suponer para el analista un esfuerzo y un gasto computacional que no quede compensado por sus bondades para la inferencia". En este trabajo se ha optado por realizar la imputación de las observaciones que faltan mediante regresión, ya que es un método que presenta buenos resultados y tiene una implementación más sencilla, tal y como señalan Gómez, Palarea y Martín (2006).

En el cuadro 1 se muestran los niveles de desigualdad para los países de América Latina obtenidos mediante el índice de Theil para el período 2004-2013. Los valores que aparecen acompañados de asteriscos han sido estimados. Con el objeto de analizar la adecuación de las estimaciones realizadas se han calculado los coeficientes de determinación y se puede apreciar que son relativamente elevados.

Cuadro 1

América Latina: índice de desigualdad de Theil, 2004-2013a

\begin{tabular}{lccccccccccc}
\hline País & 2004 & 2005 & 2006 & 2007 & 2008 & 2009 & 2010 & 2011 & 2012 & 2013 & Media \\
\hline Argentina & 0,451 & 0,449 & 0,446 & 0,423 & 0,389 & 0,358 & 0,346 & 0,332 & 0,312 & 0,310 & 0,382 \\
\hline $\begin{array}{l}\text { Bolivia (Estado } \\
\text { Plurinacional de) }\end{array}$ & $0,602^{*}$ & 0,547 & 0,472 & 0,469 & 0,389 & 0,416 & $0,34^{*}$ & 0,277 & 0,297 & 0,309 & 0,397 \\
\hline Brasil & 0,647 & 0,647 & 0,629 & 0,603 & 0,588 & 0,578 & $0,576^{*}$ & 0,560 & 0,581 & $0,543^{*}$ & 0,604 \\
\hline Chile & $0,607^{*}$ & 0,601 & 0,568 & $0,590^{*}$ & $0,584^{*}$ & 0,585 & $0,572^{*}$ & 0,541 & $0,561^{*}$ & 0,537 & 0,566 \\
\hline Colombia & 0,625 & 0,622 & $0,631^{*}$ & $0,630^{*}$ & 0,632 & 0,614 & 0,627 & 0,599 & 0,568 & 0,574 & 0,608 \\
\hline Costa Rica & 0,411 & 0,399 & 0,427 & 0,466 & 0,427 & 0,474 & 0,455 & 0,481 & 0,481 & 0,487 & 0,451 \\
\hline Ecuador & 0,605 & 0,584 & 0,747 & 0,622 & 0,512 & 0,481 & 0,491 & 0,399 & 0,430 & 0,440 & 0,531 \\
\hline El Salvador & 0,415 & 0,437 & 0,404 & 0,412 & 0,434 & 0,408 & 0,358 & 0,337 & 0,335 & 0,409 & 0,395 \\
\hline Guatemala & 0,406 & $0,483^{*}$ & 0,550 & $0,608^{*}$ & $0,657^{*}$ & $0,696^{*}$ & $0,726^{*}$ & 0,746 & $0,757^{*}$ & $0,759^{*}$ & 0,567 \\
\hline México & 0,588 & 0,635 & 0,527 & $0,559^{*}$ & 0,599 & $0,529^{*}$ & 0,458 & $0,498^{*}$ & 0,503 & $0,468^{*}$ & 0,552 \\
\hline Panamá & $0,534^{*}$ & $0,511^{*}$ & $0,547^{*}$ & $0,520^{*}$ & 0,522 & 0,532 & 0,540 & 0,557 & 0,536 & 0,522 & 0,535 \\
\hline Paraguay & 0,630 & 0,560 & 0,724 & 0,665 & 0,570 & 0,505 & 0,636 & 0,589 & 0,484 & 0,464 & 0,583 \\
\hline Perú & 0,508 & 0,522 & 0,519 & 0,516 & 0,451 & 0,432 & 0,403 & 0,387 & 0,376 & 0,367 & 0,448 \\
\hline República & 0,577 & 0,494 & 0,564 & 0,462 & 0,493 & 0,471 & 0,411 & 0,435 & 0,392 & 0,429 & 0,473 \\
Dominicana & & & & & & & & & & & \\
\hline Uruguay & 0,420 & 0,383 & 0,416 & 0,429 & 0,400 & 0,407 & 0,380 & 0,342 & 0,299 & 0,312 & 0,379 \\
\hline Media & 0,535 & 0,525 & 0,545 & 0,532 & 0,510 & 0,499 & 0,488 & 0,472 & 0,461 & 0,462 & \\
\hline Desviación típica & 0,088 & 0,082 & 0,102 & 0,082 & 0,088 & 0,088 & 0,116 & 0,124 & 0,125 & 0,115 & \\
\hline
\end{tabular}

Fuente: Elaboración propia, sobre la base de datos del Banco Mundial y la Base de Datos Socioeconómicos para América Latina y el Caribe (SEDLAC).

Nota: * $\quad$ significa que se rechaza la hipótesis nula al $10 \%$.

a Los valores de los coeficientes de determinación para cada uno de los países cuya desigualdad se ha estimado son: Bolivia (Estado Plurinacional de): 0,89; Brasil: 0,81; Chile: 0,60; Colombia: 0,84; Guatemala: 0,97; México: 0,54, y Panamá: 0,71.

En términos generales, se puede decir que, en promedio, los países con mayor nivel de desigualdad son el Brasil, Chile, Colombia, el Ecuador, Guatemala, México, Panamá y el Paraguay. En tanto, la Argentina, Bolivia (Estado Plurinacional de), Costa Rica, El Salvador, el Perú, la República Dominicana y el Uruguay presentan, en media, menores niveles de inequidad. Estos resultados coinciden, en buena medida, con los estudios de Martín (2008), Amarante, Galván y Mancero (2016), y Amarante y Jiménez (2016), entre otros.

\footnotetext{
4 La imputación de la media resulta, en términos generales, un procedimiento poco recomendable, puesto que tiene un comportamiento muy inestable.
} 
Al analizar la desigualdad de los países a lo largo del período considerado se puede determinar que existen tres grupos. Hay un primer colectivo de países -integrado por el Brasil, Chile, Colombia, México y el Paraguay - en los que la desigualdad siempre está por encima de la media. A estos países podríamos calificarlos como los más desiguales desde el punto de vista estructural, ya que su nivel de inequidad se encuentra por encima de la media en todos los años considerados. Existe un segundo grupo de países - compuesto por la Argentina, El Salvador, el Perú y el Uruguay - cuya desigualdad está, a lo largo de todo el período, por debajo de la media; estos son los países menos desiguales desde el punto de vista estructural. Por último, hay un tercer grupo de países - compuesto por Bolivia (Estado Plurinacional de), Costa Rica, el Ecuador, Guatemala, Panamá y la República Dominicana- que en algunos años presentan un índice de desigualdad por encima de la media y en otros se ubican por debajo. Estos últimos son aquellos países cuyo nivel de desigualdad va variando de acuerdo con las circunstancias económicas y sociales.

\section{La tendencia de la desigualdad}

En el cuadro 1 se puede apreciar que la desigualdad global media se va reduciendo en los últimos años. Si se consideran individualmente los índices de desigualdad de cada país en el año inicial y final del período analizado se puede observar que solo Costa Rica y Guatemala presentan mayores niveles de desigualdad al final que al principio del período; el resto de los países registran disminuciones en el período de estudio. Entre 2004 y 2013 se aprecia, entonces, una tendencia a la reducción de la desigualdad que ha sido constatada por diferentes autores, como Amarante, Galván y Mancero (2016), y Lustig, López-Calva y Ortiz-Juárez (2013). Sin embargo, algunos autores, como Piketty (2014), señalan que la desigualdad en los países latinoamericanos está subestimada por la ocultación de rentas elevadas.

Para comprobar si las diferencias entre los indicadores de desigualdad al principio y al final del período son significativas, se realiza el test de Wilcoxon para dos muestras. Los resultados se recogen en el cuadro 2.

Cuadro 2

Test de Wilcoxon, estadísticos de pruebaa

\begin{tabular}{lr}
\hline$Z$ & $-2,272$ \\
\hline Significación asintótica (bilateral) & 0,023
\end{tabular}

Fuente: Elaboración propia.

a Prueba de Wilcoxon de los rangos con signo.

La hipótesis nula del test de Wilcoxon (la desigualdad en ambos períodos coincide) se rechaza puesto que el nivel crítico es inferior al 0,05 y, por lo tanto, se detectan diferencias estadísticamente significativas en la inequidad en ambos momentos de tiempo. Por otra parte, el signo del estadístico Z muestra y corrobora la disminución en el nivel de desigualdad.

Además, se calcularon las tasas de variación de la desigualdad, tomando como momento inicial el año 2004 y como momento final el año 2013. Dado que en términos generales la desigualdad varía relativamente poco cada año, parece más adecuado para detectar cambios en los niveles de esta variable calcular dicha tasa a lo largo de todo el período de estudio. Sin embargo, y ya que en ese lapso temporal se ha producido una crisis económica a nivel mundial, también se calcularon las tasas en un punto intermedio -2007 - , por considerarlo el punto de ruptura a partir del cual se inicia la crisis. Es decir que las tasas se calcularán entre 2004 y 2007 (período anterior a la crisis) y entre 2008 y 2013 (período de crisis). Los resultados se presentan en el cuadro 3. 
Cuadro 3

Tasas de variación de la desigualdad, 2004-2013

(En porcentajes)

\begin{tabular}{lccc}
\hline País & $\mathbf{2 0 0 4 - 2 0 1 3}$ & $2004-2007$ & $2008-2013$ \\
\hline Argentina & $-31,33$ & $-6,12$ & $-20,41$ \\
\hline Bolivia (Estado Plurinacional de) & $-48,57$ & $-22,08$ & $-20,51$ \\
\hline Brasil & $-16,04$ & $-6,84$ & $-7,58$ \\
\hline Chile & $-11,53$ & $-2,87$ & $-8,02$ \\
\hline Colombia & $-8,16$ & 0,80 & $-9,18$ \\
\hline Costa Rica & 18,49 & 13,38 & 14,05 \\
\hline Ecuador & $-27,23$ & 2,81 & $-13,93$ \\
\hline El Salvador & $-1,49$ & $-0,75$ & $-5,73$ \\
\hline Guatemala & 86,95 & 49,75 & 15,53 \\
\hline México & $-20,41$ & $-4,93$ & $-21,87$ \\
\hline Panamá & $-2,25$ & $-2,62$ & 0,00 \\
\hline Paraguay & $-26,26$ & 5,55 & $-18,55$ \\
\hline Perú & $-27,80$ & 1,58 & $-18,67$ \\
\hline República Dominicana & $-25,52$ & $-19,95$ & $-12,93$ \\
\hline Uruguay & $-25,66$ & 2,21 & $-22,05$
\end{tabular}

Fuente: Elaboración propia.

Si se observa el período de estudio en su totalidad, se aprecia que la mayoría de los países han logrado disminuir su nivel de desigualdad, con la excepción de Costa Rica y Guatemala. Los países en los que más se ha reducido la desigualdad son Bolivia (Estado Plurinacional de), la Argentina, el Perú y el Ecuador. Si se consideran ambos subperíodos, en el primero se empieza a notar una tendencia a la disminución de la desigualdad que no se ve frenada por la crisis, sino todo lo contrario, la desigualdad disminuye con más fuerza, en términos generales. Este comportamiento, ya señalado por Lustig, López-Calva y Ortiz-Juárez (2013) y Cornia (2012 y 2014), entre otros, muestra que la crisis mundial no incidió en la tendencia descendente de la desigualdad, probablemente porque dicha crisis ha afectado menos a esta región que a otras regiones del mundo, como señalan Mancha, Perticarari y Buchieri (2011) y Quenan (2013).

\section{Cuantificación de los componentes de la desigualdad en América Latina}

El índice de Theil permite cuantificar la desigualdad en toda una región y dicho nivel está determinado por dos aspectos: la desigualdad dentro de cada país (desigualdad interna o DI) y la desigualdad de un país con respecto a los demás (desigualdad entre países o $D E$ ). La aplicación de la propiedad de la descomponibilidad permite conocer y medir el peso de ambos términos (véanse Villaverde (1996), Duro (2004), Goerlich y Mas (2004) y Martín (2008), entre otros). La expresión (2) se puede descomponer en dos sumandos: el primero recoge la desigualdad interna de los países y el segundo recoge la discrepancia entre los distintos países, de manera que la desigualdad total $(D T)$ es la suma de ambas, es decir:

$$
D T=D I+D E
$$

Aplicando la formulación anterior a los datos de los que se dispone, se ha cuantificado la desigualdad en toda la región constituida por los 15 países estudiados y se ha desagregado dicho valor en las componentes de desigualdad interna y entre países. Los resultados relativos al período considerado aparecen recogidos en el cuadro $4^{5}$.

\footnotetext{
5 Se han considerado los datos de la población y del ingreso mediano proporcionados por la Comisión Económica para América Latina y el Caribe (CEPAL) y la Base de Datos Socioeconómicos para América Latina y el Caribe (SEDLAC).
} 
Cuadro 4

Descomposición de la desigualdad obtenida con el índice de Theil, 2004-2013

\begin{tabular}{lccccc}
\hline Año & Desigualdad interna (DI) & $\begin{array}{c}\text { Desigualdad entre } \\
\text { países (DE) }\end{array}$ & Desigualdad total (DT) & $\begin{array}{c}\text { DE/DT } \\
\text { (en porcentajes) }\end{array}$ & $\begin{array}{c}\text { DI/DT } \\
\text { (en porcentajes) }\end{array}$ \\
\hline 2004 & 0,585 & 0,005 & 0,590 & 99,15 & 0,85 \\
\hline 2005 & 0,595 & 0,005 & 0,599 & 99,21 & 0,79 \\
\hline 2006 & 0,569 & 0,007 & 0,577 & 98,73 & 1,27 \\
\hline 2007 & 0,562 & 0,006 & 0,568 & 98,89 & 1,11 \\
\hline 2008 & 0,555 & 0,004 & 0,559 & 99,34 & 0,66 \\
\hline 2009 & 0,529 & 0,004 & 0,534 & 99,21 & 0,79 \\
\hline 2010 & 0,507 & 0,006 & 0,513 & 98,93 & 1,07 \\
\hline 2012 & 0,502 & 0,005 & 0,507 & 99,07 & 0,93 \\
\hline 2013 & 0,505 & 0,005 & 0,510 & 99,11 & 0,89 \\
\hline
\end{tabular}

Fuente: Elaboración propia.

La desigualdad global de la renta en el conjunto de América Latina ha ido disminuyendo desde 2004 hasta 2013, como ya se ha señalado. Este decrecimiento de la desigualdad se debe, sobre todo, a la disminución de las disparidades internas de los países, puesto que la desigualdad entre los países se ha mantenido estable en el período considerado. También se puede constatar que el peso del componente de desigualdad interna dentro de la desigualdad global es muy superior al de la desigualdad entre países en ese período. Estos resultados parecen indicar que el origen de la desigualdad global en la región radica tanto a las diferencias entre países, sino en la inequidad dentro de cada uno de ellos. Estos resultados son coherentes con los obtenidos por Milanovic y Muñoz (2008) que señalan que "cuando se considera América Latina como un todo, las diferencias entre las rentas medias de los países explican una parte relativamente pequeña de la desigualdad, al tiempo que la mayor parte se explica por las desigualdades existentes dentro de cada país".

Por otra parte, si se tiene en cuenta el segundo sumando $(D E)$ en la expresión (2)

$$
D E=\sum_{P=1}^{15} \frac{E_{P}(X)}{E(X)} \frac{N_{P}}{N} \log \left(\frac{E_{P}(X)}{E(X)}\right)
$$

y se analiza, se puede saber qué países son los que generan desigualdad y se "benefician" de ella y cuáles son los que la "sufren". Si $E_{P}(X)$ es inferior a $E(X)$, es decir, la renta esperada de un país está por debajo de la renta esperada de la región, el cociente es inferior a la unidad y el sumando correspondiente a ese país es negativo, se trataría de un país que "sufre" la desigualdad. Por otra parte, si $E_{P}(X)$ es superior a la renta per cápita global, el cociente es mayor que la unidad y el sumando correspondiente es positivo, se trata de un país generador de desigualdad, que se "beneficia" de ella.

En el cuadro 5 se presentan los valores de los diferentes sumandos de la desigualdad entre países.

Se aprecian comportamientos estables en el tiempo. El Brasil y Colombia, por ejemplo, son países que sufren la desigualdad, ya que su renta se ubica por debajo de la media de la región, mientras que la Argentina, Chile, Costa Rica, El Salvador, México, el Perú, la República Dominicana, el Uruguay y Venezuela (República Bolivariana de) son territorios generadores de desigualdad, ya que su renta está sobre la media.

Una vez que se ha constatado el descenso experimentado por la desigualdad en América Latina, se procederá a analizar qué determinantes pueden influir en esta evolución. Para ello, y dada la información disponible, se aplicará un análisis econométrico de datos de panel. 
Cuadro 5

Sumandos de la desigualdad entre países, 2004-2013

\begin{tabular}{lrrrrrrrrrr}
\hline País & 2004 & 2005 & 2006 & 2007 & 2008 & 2009 & 2010 & 2011 & 2012 & 2013 \\
\hline Argentina & 0,005 & 0,008 & 0,011 & 0,012 & 0,012 & 0,013 & 0,013 & 0,015 & 0,016 & 0,016 \\
\hline $\begin{array}{l}\text { Bolivia (Estado } \\
\text { Plurinacional de) }\end{array}$ & 0,000 & $-0,003$ & $-0,003$ & $-0,002$ & 0,000 & 0,001 & 0,000 & 0,002 & 0,001 & 0,000 \\
\hline Brasil & $-0,039$ & $-0,036$ & $-0,029$ & $-0,029$ & $-0,027$ & $-0,029$ & $-0,034$ & $-0,031$ & $-0,028$ & $-0,031$ \\
\hline Chile & 0,003 & 0,003 & 0,003 & 0,003 & 0,002 & 0,001 & 0,001 & 0,001 & 0,001 & 0,001 \\
\hline Colombia & $-0,004$ & $-0,002$ & $-0,017$ & $-0,016$ & $-0,010$ & $-0,011$ & $-0,012$ & $-0,010$ & $-0,010$ & $-0,010$ \\
\hline Costa Rica & 0,001 & 0,002 & 0,001 & 0,001 & 0,001 & 0,000 & 0,001 & 0,000 & 0,000 & 0,000 \\
\hline Ecuador & 0,000 & 0,000 & 0,001 & $-0,001$ & 0,001 & 0,002 & 0,001 & 0,003 & 0,003 & 0,002 \\
\hline El Salvador & 0,003 & 0,002 & 0,004 & 0,004 & 0,002 & 0,002 & 0,002 & 0,003 & 0,003 & 0,003 \\
\hline Guatemala & 0,002 & 0,001 & $-0,001$ & 0,000 & 0,000 & $-0,001$ & $-0,001$ & $-0,001$ & $-0,002$ & $-0,002$ \\
\hline México & 0,028 & 0,023 & 0,033 & 0,026 & 0,014 & 0,018 & 0,024 & 0,013 & 0,008 & 0,014 \\
\hline Panamá & $-0,001$ & 0,000 & $-0,001$ & 0,000 & 0,000 & 0,000 & 0,000 & 0,000 & $-0,001$ & 0,000 \\
\hline Paraguay & 0,001 & 0,001 & 0,000 & 0,001 & 0,001 & 0,001 & 0,000 & $-0,001$ & 0,001 & 0,000 \\
\hline Perú & 0,004 & 0,002 & 0,002 & 0,001 & 0,005 & 0,005 & 0,007 & 0,007 & 0,007 & 0,008 \\
\hline República Dominicana & 0,002 & 0,003 & 0,001 & 0,003 & 0,002 & 0,002 & 0,002 & 0,002 & 0,003 & 0,002 \\
\hline Uruguay & 0,002 & 0,002 & 0,001 & 0,001 & 0,001 & 0,001 & 0,001 & 0,001 & 0,002 & 0,002 \\
\hline Global región & 0,005 & 0,005 & 0,007 & 0,006 & 0,004 & 0,004 & 0,006 & 0,005 & 0,005 & 0,005 \\
\hline
\end{tabular}

Fuente: Elaboración propia.

\section{Determinantes de la evolución de la desigualdad de renta en países de América Latina, modelo econométrico de datos de panel}

Como ya se ha señalado, el problema de la desigualdad en América Latina se abordó en diferentes trabajos y desde distintas perspectivas. Aquí se intenta, a partir de la aplicación de una metodología de datos de panel, explorar qué variables o factores pueden determinar la evolución del nivel de desigualdad de renta en el período considerado.

Entre las variables que pueden determinar la desigualdad económica, la literatura es unánime en considerar magnitudes relativas al crecimiento económico, la educación, la sanidad o el papel de las políticas públicas, entre otras.

En concreto, en el cuadro 6 se presentan las variables empleadas en el análisis: el PIB per cápita, el gasto en sanidad per cápita, la presión fiscal, un indicador de pobreza, la tasa de alfabetización, los años dedicados al estudio y un indicador del efecto de la crisis económica.

Para la estimación del modelo se utiliza la metodología de los datos de panel, puesto que permite controlar los efectos inobservables específicos de cada país.

Las políticas públicas actúan sobre la distribución generada por el mercado a partir de instrumentos como los impuestos y las transferencias, que tienen una incidencia directa en la distribución de la renta disponible de las familias. Otra faceta de la acción redistributiva del Estado tiene lugar a través de mecanismos que pueden considerarse indirectos, como el gasto público en educación o en salud que, aunque no afectan a la renta disponible actual de los hogares, sí producen un impacto muy relevante, aunque diferido en el tiempo, en la medida en que promueven las capacidades humanas, facilitan la inserción futura en el mercado de trabajo y contribuyen a una mejor calidad de vida (Amarante y Jiménez, 2016). 
Cuadro 6

Taxonomía de las variables utilizadas

\begin{tabular}{|c|c|c|c|}
\hline & Definición & Unidades & Fuente \\
\hline Índice de Theil & Indicador de desigualdad de renta & $0-1$ & $\begin{array}{l}\text { Banco Mundial/ Base de Datos } \\
\text { Socioeconómicos para América } \\
\text { Latina y el Caribe (SEDLAC) }\end{array}$ \\
\hline PIB per cápita & $\begin{array}{l}\text { Variable sustitutiva (proxy) del } \\
\text { nivel de desarrollo de un país }\end{array}$ & Miles de dólares per cápita & CEPAL \\
\hline Gasto en sanidad per cápita & $\begin{array}{l}\text { Gasto realizado por el sector } \\
\text { público en sanidad per cápita }\end{array}$ & Miles de dólares per cápita & CEPAL \\
\hline Presión fiscal & $\begin{array}{l}\text { Ingresos impositivos en } \\
\text { relación con el PIB }\end{array}$ & Porcentajes & CEPAL \\
\hline Años de estudio & $\begin{array}{l}\text { Promedio de años de escolaridad } \\
\text { de la población de } 25 \text { a } 59 \text { años }\end{array}$ & Años & Banco Mundial/ SEDLAC \\
\hline Tasa de alfabetización & $\begin{array}{l}\text { Tasa de alfabetización de la } \\
\text { población de entre } 15 \text { y } 24 \text { años }\end{array}$ & Porcentajes & Banco Mundial/ SEDLAC \\
\hline Pobreza & Población en situación de pobreza & Porcentajes & Banco Mundial/ SEDLAC \\
\hline Efecto de la crisis económica & $\begin{array}{l}\text { Variable ficticia (dummy) (toma el } \\
\text { valor } 0 \text { en el período 2004-2007 } \\
\text { y de } 1 \text { en el período 2008-2013) }\end{array}$ & 0 y 1 & Elaboración propia \\
\hline
\end{tabular}

Fuente: Elaboración propia.

En este mismo sentido, estudios empíricos han demostrado que la educación es una herramienta que permite reducir la inequidad en el reparto de la renta (Alonso, 2001; Moller, Alderson y Nielsen, 2009; Peters, 2013, entre otros).

El papel de la política fiscal en la explicación de la desigualdad ha sido puesto de manifiesto por autores como Mercader-Prats y Levy (2004), Atkinson y Brandolini (2009), Muinelo y Roca (2013). En este sentido, la estructura del sistema impositivo debería jugar un papel muy relevante (Molina, Guarnido y Amate, 2013). Peters (2013) señala que la política fiscal es una variable endógena que refleja, a través de procesos políticos, las preferencias de los ciudadanos (votantes) por la distribución de la renta.

Levy y Schady (2013) y Azevedo, Inchaust y Sanfelice (2013) señalan una relación directa entre desigualdad económica y pobreza, ambas aumentan y disminuyen de acuerdo con unas pautas similares.

Sin embargo, desde que Kuznets (1955) propusiera su conocida "hipótesis U”, el crecimiento económico ha sido el factor más estudiado como variable explicativa de la desigualdad en la distribución de la renta. Los efectos de esta variable sobre la desigualdad, no obstante, no son unánimes. En el contexto de los países de América Latina, De Janvry y Sadoulet (2000) y Medina y Galván (2014a y 2014b) han abordado las relaciones entre crecimiento económico, desigualdad y pobreza.

\section{Modelo de datos de panel, especificación econométrica}

Esta metodología permite contemplar la existencia de efectos latentes no observables, específicos de cada país (efectos individuales). Si estos efectos no observables existen y no se corrigen, se producirá un problema de variables omitidas y los coeficientes estimados en el modelo estarán sesgados (véase una descripción detallada de la metodología de datos de panel en Baltagi, 1995). Además, para evitar el potencial sesgo de variable omitida por las variables que van cambiando con el tiempo, pero que son constantes entre países, en el modelo se incluyen efectos temporales. 


$$
I T(X)_{i t}=\alpha_{i}+\beta_{0}+\beta_{1} P I B p c_{i t}+\beta_{2} G S_{i t}+\beta_{3} I P_{i t}+\beta_{4} P F_{i t}+\beta_{5} T A_{i t}+\beta_{6} A E_{i t}+\beta_{7} E C_{i t}+u_{i t}
$$

Donde $i$ denota a los países considerados $(i=1, \ldots, 15), t$ son los años $(t=2004, \ldots, 2013)$, $I T$ es el índice de Theil, PIBpc es el PIB per cápita, $G S$ es el gasto en sanidad, $A E$ son los años de escolaridad, $T A$ es la tasa de alfabetización, $P F$ es la presión fiscal, $I P$ es un índice de pobreza y $E C$ es una variable que recoge el efecto de la crisis, la cual toma el valor cero de 2004 a 2007 y el valor uno de 2008 a $2013^{6}$. $\alpha_{i}$ representa el efecto individual específico de cada país, que se incluye en el modelo para tener en cuenta cualquier factor que pueda influir en el nivel de desigualdad más allá de las variables explicativas consideradas en el modelo. Las perturbaciones se denotan mediante $u_{i t}$ y se asumen como independientes e idénticamente distribuidas, con media cero y varianza $\sigma_{u}^{2}$.

Con el fin de identificar la especificación del modelo de panel más adecuado, se ha realizado la estimación teniendo en cuenta tanto un modelo de efectos fijos como uno de efectos aleatorios. El modelo de efectos fijos considera cada $\alpha_{i}$ como una constante en la regresión, mientras que el de efectos aleatorios considera a $\alpha_{i}$ como una componente de la perturbación aleatoria. Con el fin de establecer si el estimador de efectos aleatorios es más apropiado que el de efectos fijos se realiza el test de Hausman (1978). Además, se contrasta la existencia de efectos específicos para cada país a partir del test $F$ (en efectos fijos) o el test de Breusch-Pagan (en efectos aleatorios). En ambos casos, la hipótesis nula es que $\alpha_{i}$ es igual para todos los países. Si no se rechaza dicha hipótesis, se trata de un modelo de regresión clásico y se puede estimar por mínimos cuadrados ordinarios. Es decir que las pruebas de Breusch y Pagan y la prueba $F$ de efectos fijos indican, respectivamente, que tanto el modelo de efectos aleatorios como el de efectos fijos son mejores que el modelo con datos agrupados o fusionados.

En el cuadro 7 se recogen los principales estadísticos descriptivos de las variables analizadas.

Cuadro 7

Principales estadísticos descriptivos de las variables empleadas

\begin{tabular}{lccccc}
\hline Variables & Media & Mediana & Mínimo & Máximo & Desviación típica \\
\hline Índice de Theil & 0,52 & 0,52 & 0,25 & 0,84 & 0,13 \\
\hline PIBpc & 6,74 & 5,98 & 1,68 & 14,29 & 3,42 \\
\hline Gasto en salud & 0,16 & 0,11 & 0,01 & 0,54 & 0,13 \\
\hline Presión fiscal & 13,37 & 13,28 & 8,11 & 20,84 & 3,03 \\
\hline Años de escolaridad & 8,66 & 8,80 & 4,30 & 11,33 & 1,56 \\
\hline Tasa de alfabetización & 97,60 & 98,33 & 84,99 & 99,75 & 2,58 \\
\hline Índice de pobreza & 6,48 & 6,02 & 0,23 & 20,71 & 4,30 \\
\hline
\end{tabular}

Fuente: Elaboración propia.

\section{Resultados empíricos}

Los resultados de las estimaciones finales del modelo de datos de panel aparecen recogidos en el cuadro 8. Se ha realizado el test de Hausman y se obtuvo un valor del estadístico Chi-cuadrado $(7)=4,33$ con un nivel crítico de $p=0,7406$, con lo que se concluye que el modelo de efectos aleatorios es el adecuado.

\footnotetext{
6 Se ha considerado una situación de crisis cuando se produce una caída de la tasa de crecimiento del PIB. En este trabajo se hace referencia a la crisis mundial que se registró en el período 2008-2013.
} 


\section{Cuadro 8}

Modelos de datos de panel estimados para explicar la desigualdad en países latinoamericanos, 2004-2013

\begin{tabular}{|c|c|}
\hline Variables & Modelo de datos de panel \\
\hline \multirow{2}{*}{$\beta_{0}$} & $-1,9952$ \\
\hline & $(0,009)$ \\
\hline \multirow[t]{2}{*}{$\beta_{1}$ (PIB per cápita) } & $0,0226^{\star \star \star}$ \\
\hline & $(0,051)$ \\
\hline \multirow{2}{*}{$\beta_{2}$ (Gasto en sanidad per cápita) } & $-0,4372^{* \star \star}$ \\
\hline & $(0,011)$ \\
\hline \multirow[t]{2}{*}{$\beta_{3}$ (Pobreza) } & $0,0057^{\star \star \star}$ \\
\hline & $(0,096)$ \\
\hline \multirow[t]{2}{*}{$\beta_{4}$ (Presión fiscal) } & $-0,0169^{\star \star \star}$ \\
\hline & $(0,000)$ \\
\hline \multirow[t]{2}{*}{$\beta_{5}$ (Tasa de alfabetización) } & $-0,030^{\star \star \star}$ \\
\hline & $(0,000)$ \\
\hline \multirow[t]{2}{*}{$\beta_{6}$ (Años de estudio) } & $-0,0546^{\star \star *}$ \\
\hline & $(0,006)$ \\
\hline \multirow[t]{2}{*}{$\beta_{7}$ (Efecto de la crisis) } & $-0,0138$ \\
\hline & 0,329 \\
\hline Observaciones & 145 \\
\hline $\mathrm{R}^{2}$ & 0,52 \\
\hline \multirow[t]{2}{*}{ Test de Breusch-Pagan } & $x^{2}(1)=290,18^{\star \star \star}$ \\
\hline & $p \approx 0$ \\
\hline \multirow[t]{2}{*}{ Test $\mathrm{F}$} & $F(14,123)=27,23^{\star \star \star}$ \\
\hline & $p \approx 0$ \\
\hline \multirow[t]{2}{*}{ Test de Hausman } & $x^{2}(7)=4,33$ \\
\hline & $p=0,7406$ \\
\hline
\end{tabular}

Fuente: Elaboración propia.

Nota: ${ }^{\star \star \star}$ significa que se rechaza la hipótesis nula al $1 \%$.

Al centrarse en los resultados del modelo, se aprecia que los contrastes sobre los efectos individuales son significativos y a partir del test de Breusch-Pagan se rechaza la correspondiente hipótesis nula sobre la inexistencia efectos individuales.

Respecto de los efectos individuales (dada su existencia), los estadísticos F del contraste indican que no se rechaza la hipótesis nula (no hay efectos temporales) y, por tanto, no es adecuado incorporar tales efectos en el modelo.

Considerando los resultados del cuadro 8 se puede apreciar que todas las variables, a excepción del efecto de la crisis, son significativas al 1\%. Este resultado parece indicar que la crisis económica no ha tenido efectos significativos en América Latina, o al menos no los ha tenido en relación con la evolución de la desigualdad económica.

Con respecto al desarrollo económico, medido a partir del PIB, se aprecia una relación positiva, es decir, un mayor desarrollo económico del país conduce a un aumento de la desigualdad. Este resultado es coherente con los obtenidos por Ravallion y Chen (1997), Molina, Amate y Guarnido (2011), y Acar y Dogruel (2012), para quienes el crecimiento económico no reduce las desigualdades de renta. Empleando el PIB per cápita como variable sustitutiva (proxy) del desarrollo económico, Molina, Amate y Guarnido (2011) encuentran que este aumenta la inequidad en los países de la Unión Europea. Este comportamiento parece coincidir con las críticas que se efectúan al PIB como medida de desarrollo de un país, ya que solo considera ciertos aspectos económicos y deja fuera comportamientos sociales y medioambientales. Así, un aumento global del PIB en un país puede indicar crecimiento, pero no tiene por qué mostrar un crecimiento equilibrado (Costanza y otros, 2009). 
Entre las variables que pueden determinar la desigualdad, en la literatura se da cuenta de que la educación es una herramienta que permite reducirla (Alonso, 2001; Moller, Alderson y Nielsen, 2009; Peters, 2013, entre otros), por lo que la alfabetización y los años dedicados al estudio ejercen un efecto redistributivo (Molina, Guarnido y Amate, 2013). América Latina ha ampliado considerablemente la cobertura básica de educación: el porcentaje de niños que están inscritos en el nivel adecuado para su edad supera el $90 \%$ en la escuela primaria y se ubica entre el $60 \%$ y el $80 \%$ en la escuela secundaria en la mayoría de los países de la región (Levy y Schady, 2013).

El efecto de la presión fiscal sobre la distribución de la renta es el esperado, aspecto que ha sido estudiado por Itriago (2014). Sin embargo, algunos autores señalan que la capacidad de redistribución por medio de impuestos y transferencias es limitada para alterar los niveles de desigualdad en el acceso a recursos de los hogares (Amarante y Jiménez, 2016). Esta menor capacidad tiene que ver con los bajos niveles de ingresos tributarios y su menor impacto distributivo. En muchos casos, el aumento del gasto público redistributivo se financió a partir de impuestos regresivos, por lo que los efectos netos de la política fiscal se vieron notablemente reducidos (Gómez Sabaini y Morán, 2013).

Otra de las variables que genera una corrección de los niveles de desigualdad es el gasto en salud. Un aumento de dicha variable conduce, de una manera indirecta, a la reducción de la desigualdad, ya que afecta las decisiones y posibilidades de consumo, en la medida en que la disponibilidad de servicios públicos de salud de calidad puede liberar recursos de las familias para destinar a otros consumos (Gómez Sabaini y Morán, 2013; Atun y otros, 2015). En este sentido, el signo del coeficiente de la variable asociada a la sanidad es el esperado.

El coeficiente que acompaña a la variable pobreza también es coherente con la relación directa que numerosos autores habían indicado que tiene con la desigualdad (Levy y Schady, 2013; Azevedo, Inchaust y Sanfelice, 2013). La pobreza ha disminuido durante el período considerado, lo que probablemente ha repercutido en un descenso del nivel de inequidad (Cruces y Gasparini, 2013; Gasparini y Gluzmann, 2012).

Se puede entonces concluir que la reducción en los niveles de desigualdad experimentados en América Latina se debe, al menos en parte, a un descenso de la pobreza en los países, así como a un intento de incrementar y generalizar las políticas redistributivas directas e indirectas ${ }^{7}$.

\footnotetext{
7 En este trabajo se ha utilizado como indicador de desigualdad el índice de Theil, cuya información se obtuvo de los datos proporcionados por el Banco Mundial y el Centro de Estudios Distributivos, Laborales y Sociales (CEDLAS). Entre otros motivos, se utilizó la Base de Datos Socioeconómicos para América Latina y el Caribe (SEDLAC) porque proporciona información sobre la Argentina, lo que no ocurre con otras bases de datos consultadas. Este aspecto se considera relevante dado el peso que este país tiene en la economía de América Latina (en 2015 representó aproximadamente un 11\% del PIB de América Latina y Caribe). Además, hay otros países, como Bolivia (Estado Plurinacional de), El Salvador y el Perú, donde es aconsejable el uso de la base de datos del Banco Mundial, dada la falta significativa de datos en otras fuentes. Sin embargo, también se considera que la CEPAL publica una base de datos muy bien fundamentada en la que se cuantifica la desigualdad mediante, entre otros, el índice de Theil (véase CEPALSTAT [en línea] http://estadísticas.cepal.org/cepalstat). A modo de ilustración se han realizado algunas comparaciones entre los resultados generados a partir de la aplicación de ambas bases para tener una medida de la consistencia de las conclusiones obtenidas en este trabajo. Inicialmente se ha realizado un contraste no paramétrico de Mann-Whitney-Wilcoxon sobre la variable índice de Theil para cada país, con el objetivo de determinar si hay comportamientos similares entre los índices proporcionados por ambas bases (CEPAL y Banco Mundial). De la aplicación de este test se desprende el no rechazo de la hipótesis nula (comportamientos similares de los indicadores de las dos fuentes estadísticas) con niveles críticos por encima del 0,05 para todos los países analizados (a excepción del Brasil y la República Dominicana, para los que se rechazaría la hipótesis). A continuación, y siguiendo con esta comparación, se ha estimado el modelo de datos de panel con los datos del índice de Theil de la CEPAL y se obtuvieron comportamientos similares para el conjunto de variables, tanto en lo que se refiere a los signos de los coeficientes como a su escala. Todo ello parece conducir a que los resultados son robustos respecto de la base de datos empleada para cuantificar los indicadores de desigualdad.
}

$I T(X)_{i t}=2,689+0,00001 * * P I B p c_{i t}-0,0212 * * G S_{i t}+0,0077 I P_{i t}-0,0002 * * P F_{i t}-0,0073^{* *} T A_{i t}-0,1056^{* *} A E_{i t}-0,0002 E C_{i t}+u_{i t}$

Donde ** indica que las estimaciones son significativamente distintas de cero, por debajo del nivel del $5 \%$. En lo que se refiere a los datos de pobreza, que en cierta medida también puede indicar los desequilibrios en el reparto de la renta, los datos que informa la CEPAL en relación con esta variable se toman del Banco Mundial, por lo que no tiene sentido realizar ninguna comparación. 


\section{Posicionamiento de países de América Latina de acuerdo con la reducción de sus niveles de desigualdad}

En el apartado $V$ se han determinado, a partir de la utilización de una técnica econométrica de datos de panel, algunas de las variables significativas que permiten explicar, al menos en parte, la reducción de la desigualdad en América Latina. Mediante el empleo de dichas variables y la aplicación de una técnica multivariante de clasificación se agrupará a los países objeto de estudio y se determinará cuáles son los que se encuentran en mejores condiciones para reducir su nivel de desigualdad.

Inicialmente se efectuará un análisis clúster jerárquico con el objetivo de clasificar los países de acuerdo con su disposición para reducir la desigualdad. El método aplicado para establecer los grupos es el de Ward y la medida de distancia empleada es la euclídea al cuadrado. Dicha técnica se aplicará sobre las variables que se han estimado como relevantes en la reducción de la inequidad (PIB per cápita, pobreza, gasto sanitario, años de escolaridad, tasa de alfabetización y presión fiscal) referentes al último año del período considerado (2013). No obstante, con el objeto de probar la robustez de los resultados así obtenidos, se ha repetido el análisis para el resto de los años y se han obtenido resultados similares.

A partir de la aplicación de dicha técnica se pueden detectar tres clústers o conglomerados (véase el cuadro 9).

Cuadro 9

Agrupaciones de países de acuerdo con factores de reducción de la desigualdad

\begin{tabular}{lll}
\hline Clúster 1 & Clúster 2 & Clúster 3 \\
\hline Argentina & Bolivia (Estado Plurinacional de) & Guatemala \\
\hline Brasil & Colombia & \\
\hline Chile & Ecuador & \\
\hline Costa Rica & El Salvador & \\
\hline México & Paraguay & \\
\hline Panamá & Perú & \\
\hline Uruguay & República Dominicana & \\
\hline
\end{tabular}

Fuente: Elaboración propia.

Puede resultar extraño que en uno de los grupos haya un único país, pero se ha decidido mantener esta estructura de tres conglomerados dada la especial idiosincrasia de Guatemala, que hace que este país sea muy diferente del resto.

El primer conglomerado está constituido por la Argentina, el Brasil, Chile, Costa Rica, México, Panamá y el Uruguay, el segundo está formado por Bolivia (Estado Plurinacional de), Colombia, el Ecuador, El Salvador, el Paraguay, el Perú y la República Dominicana, y el tercero incluye solo a Guatemala.

En el cuadro 10 se caracterizan dichos grupos a partir de las variables iniciales. Como puede observarse en el cuadro 10, el primer clúster está constituido por aquellos países con menor tasa de pobreza y mayores niveles de PIB per cápita, gasto en salud, tasa de alfabetización y años de estudio, en media. Es posible señalar que este conjunto de países está en mejores condiciones para continuar disminuyendo los niveles de desigualdad (si se consideran exclusivamente las variables relevantes 
surgidas del análisis). El grupo 2 está conformado por países con mayores niveles de pobreza que el anterior y menores valores del resto de magnitudes analizadas, en promedio. Por ello, se considera que estos países estarán en peores condiciones que los del primer grupo para disminuir los niveles de desigualdad. Por último, Guatemala presenta las mayores tasas de pobreza y los menores niveles de PIB per cápita, gasto en salud, tasa de alfabetización y años de estudio, en promedio, con lo que se puede concluir que sería el país peor posicionado para reducir la tasa de desigualdad (si solo se consideran las variables objeto de estudio).

Cuadro 10

Descripción de los conglomerados ${ }^{a}$

\begin{tabular}{llrrrr}
\hline & N & Mínimo & Máximo & Media & Desviación estándar \\
\hline 1 & 7 & 0,34 & 6,01 & 2,6329 & 2,09523 \\
\hline Pobreza & 7 & 8,75 & 14,29 & 11,4714 & 2,13075 \\
\hline PIB per cápita & 7 & 0,08 & 0,54 & 0,3529 & 0,15892 \\
\hline Gasto en salud & 7 & 9,71 & 19,34 & 13,7886 & 3,58807 \\
\hline Presión fiscal & 7 & 8,36 & 11,33 & 9,9557 & 1,14490 \\
\hline Años de estudio & 7 & 98,16 & 99,41 & 98,8657 & 0,40435 \\
\hline Tasa de alfabetización & 7 & 2,19 & 7,70 & 4,2443 & 2,02906 \\
\hline Pobreza & 7 & 2,23 & 7,06 & 4,7714 & 1,65931 \\
\hline PIB per cápita & 7 & 0,02 & 0,13 & 0,0829 & 0,03450 \\
\hline Gasto en salud & 7 & 11,85 & 20,84 & 15,3200 & 2,83287 \\
\hline Presión fiscal & 7 & 6,56 & 10,02 & 8,9800 & 1,13561 \\
\hline Años de estudio & 7 & 97,22 & 99,44 & 98,3957 & 0,80027 \\
\hline Tasa de alfabetización & 1 & 14,49 & 14,49 & 14,4900 &. \\
\hline Pobreza & 1 & 2,92 & 2,92 & 2,9200 & \\
\hline PIB per cápita & 1 & 0,03 & 0,0300 & \\
\hline Gasto en salud & 1 & 10,75 & 10,7500 & \\
\hline Presión fiscal & 1 & $4,8,82$ & 4,8200 &. \\
\hline Años de estudio & 1 & 91,90 & 91,9000 &. \\
\hline Tasa de alfabetización & & & &. \\
\hline
\end{tabular}

Fuente: Elaboración propia.

a En el conglomerado 3, formado por un único país, no tiene sentido calcular la desviación típica.

Continuando con esta idea, se ha construido un indicador sintético, utilizando la metodología del análisis factorial multivariante, que permita establecer una clasificación de países de acuerdo con su posición para disminuir el nivel de inequidad. Este índice se ha determinado para 2013. Si bien las autoras son conscientes de que el número de variables que se usaron para la construcción de este indicador sintético es muy reducido, han considerado que la realización de este análisis es interesante y aporta más luz sobre el fenómeno estudiado.

A partir de la aplicación de las pruebas de Kaiser-Meyer-Olkin (KMO) y de Barlett se puede concluir que los datos se adecúan a una modelización factorial. En el cuadro 11 se aprecia que la prueba KMO está próxima a 0,7 y el test de Barlett resulta significativo para rechazar la hipótesis nula.

Cuadro 11

Pruebas de Kaiser-Meyer-Olkin (KMO) y de Barlett

\begin{tabular}{lcr}
\hline Medida Kaiser-Meyer-Olkin & & 0,682 \\
\hline Prueba de esfericidad de Bartlett & Aprox. Chi-cuadrado & 47,002 \\
\cline { 2 - 3 } & gl & 15 \\
\cline { 2 - 3 } & Sig. & 0,000 \\
\hline
\end{tabular}

Fuente: Elaboración propia. 
De la aplicación del análisis factorial se deduce que las variables consideradas quedan bien explicadas por los factores retenidos, pues todas las comunalidades están por encima de 0,6 (entre ellas se destaca el PIB per cápita). Las comunalidades se determinan a partir de la suma de los coeficientes de correlación al cuadrado de cada variable con el conjunto de factores retenidos

$$
h^{2}(m)=r_{x j, y l}^{2}+\ldots+r^{2}{ }_{X j, Y m}
$$

donde $Y_{i}$ representa al factor $i$-ésimo y $X_{j}$ a la variable $j$-ésima, y $m$ es el número de factores extraídos (véase el cuadro 12).

Cuadro 12

Comunalidades ${ }^{\mathrm{a}}$

\begin{tabular}{lcc}
\hline & Inicial & Extracción \\
\hline Pobreza & 1,000 & 0,753 \\
\hline PIB per cápita & 1,000 & 0,864 \\
\hline Gasto en salud & 1,000 & 0,793 \\
\hline Presión fiscal & 1,000 & 0,642 \\
\hline Años de estudio & 1,000 & 0,777 \\
\hline Tasa de alfabetización & 1,000 & 0,860 \\
\hline
\end{tabular}

Fuente: Elaboración propia.

a El método de extracción aplicado es el de componentes principales.

En el cuadro 13 se observa que la información contenida en las variables iniciales se condensa en dos factores que retienen una proporción de varianza en torno al 78\%.

Cuadro 13

Autovalores y proporción de inercia retenida

\begin{tabular}{lccc}
\hline \multirow{2}{*}{ Componente } & \multicolumn{3}{c}{ Autovalores iniciales } \\
\cline { 2 - 4 } & Total & Porcentaje de varianza & Porcentaje acumulado \\
\hline 1 & 3,483 & 58,051 & 58,051 \\
\hline 2 & 1,206 & 20,098 & 78,148 \\
\hline 3 & 0,730 & 12,158 & 90,307 \\
\hline 4 & 0,328 & 5,460 & 95,767 \\
\hline 6 & 0,163 & 2,723 & 98,490 \\
\hline
\end{tabular}

Fuente: Elaboración propia.

En el cuadro 14 se muestra la matriz de componentes rotados a partir de la aplicación del método Varimax. Se aprecia que el primer factor está relacionado directamente con el PIB per cápita y el gasto en salud e inversamente con la pobreza. Se trata de un eje que recoge factores económicos que intervienen en la disminución de la desigualdad. El segundo factor está relacionado directamente con la tasa de alfabetización, los años de estudio y los impuestos, es decir, representa el efecto de las políticas fiscales y sociales, fundamentalmente educativas, en la disminución de la desigualdad. 
Cuadro 14

Matriz de componentes rotados ${ }^{a}$

\begin{tabular}{lcc} 
& \multicolumn{3}{c}{ Componente } \\
\cline { 2 - 3 } & 1 & 2 \\
\hline PIB per cápita & 0,928 & 0,051 \\
\hline Gasto en salud & 0,890 & 0,037 \\
\hline Pobreza & $-0,668$ & $-0,553$ \\
\hline Tasa de alfabetización & 0,484 & 0,791 \\
\hline Presión fiscal & $-0,198$ & 0,776 \\
\hline Años de estudio & 0,609 & 0,637
\end{tabular}

Fuente: Elaboración propia.

a Método de rotación: Varimax con normalización Kaiser. La rotación ha convergido en tres iteraciones.

Para la elaboración de un indicador sintético se obtendrán las puntuaciones factoriales asociadas a las variables iniciales (véase el cuadro 15).

Cuadro 15

Matriz de puntuaciones

\begin{tabular}{lrc} 
& \multicolumn{2}{c}{ Componente } \\
\cline { 2 - 3 } & 1 & 2 \\
\hline Pobreza & $-0,162$ & $-0,185$ \\
\hline PIB per cápita & 0,436 & $-0,232$ \\
\hline Gasto en salud & 0,406 & $-0,203$ \\
\hline Presión fiscal & $-0,220$ & 0,423 \\
\hline Años de estudio & 0,135 & 0,216 \\
\hline Tasa de alfabetización & 0,023 & 0,370 \\
\hline
\end{tabular}

Fuente: Elaboración propia.

A partir de los valores anteriores se ha obtenido el indicador sintético (IS) para el año considerado, cuya expresión es la que sigue:

$$
I S_{j}=\sum_{i=1}^{r} z_{r j} X_{i j}
$$

Donde $z_{r j}$ representa la media ponderada de las puntuaciones factoriales y $X_{i j}$ las variables iniciales. Los resultados se muestran en el cuadro 16.

Cuadro 16

Indicador sintético de posicionamiento ante la desigualdad

\begin{tabular}{lcc}
\hline País & Indicador & Clasificación \\
\hline Chile & 12,235 & 1 \\
\hline Argentina & 12,113 & 2 \\
\hline Uruguay & 11,941 & 3 \\
\hline Panamá & 11,129 & 4 \\
\hline Costa Rica & 10,888 & 5 \\
\hline Brasil & 10,871 & 6 \\
\hline México & 10,558 & 7 \\
\hline República Dominicana & 9,967 & 8 \\
\hline Perú & 9,865 & 9 \\
\hline
\end{tabular}


Cuadro 16 (conclusión)

\begin{tabular}{lcc}
\hline País & Indicador & Clasificación \\
\hline Colombia & 9,773 & 10 \\
\hline Paraguay & 9,770 & 11 \\
\hline Ecuador & 9,730 & 12 \\
\hline El Salvador & 9,024 & 13 \\
\hline Bolivia (Estado Plurinacional de) & 8,514 & 14 \\
\hline Guatemala & 6,898 & 15 \\
\hline
\end{tabular}

Fuente: Elaboración propia.

Los resultados obtenidos a partir de la construcción del indicador sintético son coherentes con los que se derivan del análisis clúster: los países con puntuación más alta en la clasificación (Chile, Argentina, Uruguay, Panamá, Costa Rica, Brasil y México) son los que se encuentran en el primer conglomerado. Los siguientes (República Dominicana, Perú, Colombia, Paraguay, Ecuador, El Salvador y Bolivia (Estado Plurinacional de)) están en el segundo conglomerado y Guatemala está en la última posición de la clasificación y notablemente separado del país anterior.

\section{Conclusiones}

En este trabajo se estudia la desigualdad en los países de América Latina mediante el índice de Theil en el período 2004-2013. Se ha utilizado dicho indicador por las adecuadas propiedades que verifica, en particular la descomponibilidad. En los diferentes territorios se ha observado una evolución heterogénea relativa a la inequidad. Como es lógico, se obtuvieron diferencias sustanciales entre un país y otro.

Los países con una mayor desigualdad de renta en el período considerado son el Brasil, Chile, Colombia, México y el Paraguay. Entre los menos desiguales se encuentran la Argentina, El Salvador, el Perú y el Uruguay. En el resto de los países, la desigualdad va variando de acuerdo con las circunstancias económicas y sociales (tal es el caso de Bolivia (Estado Plurinacional de), Costa Rica, el Ecuador, Guatemala, Panamá y la República Dominicana).

Asimismo, se aprecia que la mayoría de los países ha visto disminuir el nivel de desigualdad, con la excepción de Costa Rica y Guatemala. Los países que más han disminuido la desigualdad en ese período son Bolivia (Estado Plurinacional de), la Argentina, el Perú y el Ecuador.

Por otra parte, se ha obtenido la desigualdad global de América Latina a partir de la desigualdad interna de los países y de la desigualdad entre países, y se ha constatado un descenso de dicha desigualdad desde 2005 hasta 2013.

Considerando el signo del indicador de desigualdad entre países, es posible determinar qué naciones sufren la desigualdad y cuáles la generan. De ello se deduce que el Brasil y Colombia sufren la desigualdad todos los años, ya que su renta se ubica por debajo de la media global. El resto de los países, en tanto, se benefician de ella, ya que su renta está sobre la media.

Con el objeto de estudiar las variables socioeconómicas determinantes del nivel de desigualdad se ha estimado un modelo de datos de panel de efectos aleatorios, donde se ha encontrado que las siguientes variables son significativas para explicar la desigualdad: PIB per cápita, gasto en sanidad per cápita, presión fiscal, tasa de pobreza, tasa de alfabetización y años de estudio. La crisis económica que se desencadenó en 2008, y que tanto ha afectado a Europa, no ha resultado significativa.

Para sistematizar el comportamiento de los países frente a la desigualdad se llevó a cabo un análisis de agrupación, empleándose para ello el algoritmo clúster jerárquico, se han obtenido tres conglomerados: uno formado por los países con niveles bajos de desigualdad, otro integrado por 
países con altos niveles de desigualdad y un tercero compuesto solo por Guatemala. Los grupos son bastante estables y se registran pocas modificaciones en su composición, con lo cual parece que la desigualdad es una característica con marcado carácter estructural.

Por último, se ha construido un indicador sintético mediante la metodología del análisis factorial, que permite establecer una clasificación de países de acuerdo con su posición para disminuir el nivel de inequidad.

\section{Bibliografía}

Acar, S. y F. Dogruel (2012), "Sources of inequality in selected MENA countries", Structural Change and Economic Dynamics, vol. 23, №3, Amsterdam, Elsevier.

Adiego, M. y L. Ayala (2013), "La estructura de la desigualdad de la renta en el largo plazo", Revista de Economía Aplicada, vol. 21, № 62, Zaragoza.

Alonso, A. (2001), "Logistic regression and world income distribution", International Advances in Economic Research, vol. 7, № 2, Springer.

Amarante,V., M. Galván y X. Mancero (2016), "Desigualdad en América Latina: una medición global”, Revista CEPAL, N 118 (LC/G.2676-P), Santiago, Comisión Económica para América Latina y el Caribe (CEPAL).

Amarante. V. y J. P. Jiménez (2016), "Distribución del ingreso e imposición a las altas rentas en América Latina", Cuadernos de Economía, vol. 35, № 67, Bogotá, Universidad Nacional de Colombia.

Ariza, M. y O. de Oliveira (2007), "Familias, pobreza y desigualdad social en Latinoamérica: una mirada comparativa", Estudios Demográficos y Urbanos, vol. 22, N1․ Ciudad de México, El Colegio de México.

Atkinson, A. B. y A. Brandolini (2009), "The panel-of-countries approach to explaining income inequality: an interdisciplinary research agenda", Mobility and Inequality: Frontiers of Research in Sociology and Economics, S. L. Morgan, D. B. Grusky y G. S. Fields (eds.), Stanford, Stanford University Press.

Atkinson, A. B. y S. Morelli (2011), "Economic crisis and inequality", Human Development Research Paper, $N^{\circ}$ 2011/06, Programa de las Naciones Unidas para el Desarrollo (PNUD).

Atun, R. y otros (2015), "La reforma de los sistemas de salud y la cobertura universal de salud en América Latina", MEDICC Review, vol. 17, № 1

Ayala, L. (2013), "Crisis económica y distribución de la renta: una perspectiva comparada", Papeles de Economía Española, № 135, Madrid, Funcas.

Azevedo, J. P., G. Inchaust y V. Sanfelice (2013), "Decomposing the recent inequality decline in Latin America", Policy Research Working Paper, № 6715, Washington, D.C., Banco Mundial.

Azevedo, J. P. y otros (2013), "Fifteen years of inequality in Latin America: how have labor markets helped?", Policy Research Working Paper, № 6384, Washington, D.C., Banco Mundial.

Baltagi, B. H. (1995), Econometric Analysis of Panel Data, Nueva York, John Wiley \& Sons.

Banco Mundial, "Datos de libre acceso del Banco Mundial" [en línea] http://datos.bancomundial.org/.

CEPALSTAT (Base de Datos y Publicaciones Estadísticas) [en línea] http://estadisticas.cepal.org/cepalstat/.

Cornia, G. A. (2014), "Income inequality in Latin America: recent decline and prospects for its further reduction", serie Macroeconomía del Desarrollo, № 149 (LC/L.3847), Santiago, Comisión Económica para América Latina y el Caribe (CEPAL).

(2012), "Inequality trends and their determinants. Latin America over 1990-2010", Documento de Trabajo, $N^{\circ}$ 2012/09, Universidad de las Naciones Unidas.

Costanza, R. y otros (2009), "Beyond GDP: the need for new measures of progress", The Pardee Papers, $N^{\circ} 4$, Boston, Universidad de Boston.

Cowell, F. A. (1977), Measuring Inequality, Oxford, Philip Allan Publishers.

Cruces, G. y L. Gasparini (2013), "Políticas sociales para la reducción de la desigualdad y la pobreza en América Latina y el Caribe. Diagnóstico, propuesta y proyecciones en base a la experiencia reciente", Documentos de Trabajo del CEDLAS, № 0142, La Plata, Centro de Estudios Distributivos, Laborales y Sociales (CEDLAS) de la Universidad Nacional de La Plata.

Dagum, C. (2001), "Desigualdad del rédito y bienestar social, descomposición, distancia direccional y distancia métrica entre distribuciones", Estudios de Economía Aplicada, vol. 17, № 1, Asociación Internacional de Economía Aplicada. 
De Janvry, A. y E. Sadoulet (2000), "Growth, poverty, and inequality in Latin America: a causal analysis, 1970-94", Review of Income and Wealth, vol. 46, N³, Wiley, septiembre.

Duro, J. A. (2004), "La descomposición de la desigualdad de renta per cápita por factores multiplicativos a través del índice de Theil: una revisión metodológica e ilustración para las provincias españolas", Revista de Estudios Regionales, N 70, Málaga, Universidad de Málaga.

Foster, J. E. (1983), "An axiomatic characterization of the Theil measure of income inequality", Journal of Economic Theory, vol. 31, № 1, Amsterdam, Elsevier.

Gasparini, L. y P. Gluzmann (2012), "Estimating income poverty and inequality from the Gallup World Poll”, Journal of Income Distribution, vol. 21, N 1, Toronto, Universidad de York.

Goerlich, F. J. y M. Mas (2004), "Distribución personal de la renta en España (1973-2001)", Papeles de Economía Española, vol. 1, №100, Madrid, Funcas.

Gómez, J., J. Palarea y J. A. Martín (2006), "Métodos de inferencia estadística con datos faltantes. Estudio de simulación sobre los efectos en las estimaciones", Estadística Española, vol. 48, № 162, Madrid, Instituto Nacional de Estadística (INE).

Gómez Sabaini, J. C. y D. Morán (2013), "Política tributaria en América Latina: agenda para una segunda generación de reformas", serie Macroeconomía del Desarrollo, №133 (LC/G.3632), Santiago, Comisión Económica para América Latina y el Caribe (CEPAL).

Hausman, J. A. (1978), "Specification tests in econometrics", Econometrica, vol. 46, № 6, Nueva York, The Econometric Society.

Itriago, D. (2014), "Política fiscal y desarrollo en América Latina y Caribe", Economía Exterior, № 70.

Kuznets, S. (1955), "Economic growth and income inequality", The American Economic Review, vol. 45, $\mathrm{N}^{\circ}$ 1, Nashville, Tennessee, American Economic Association.

Levy, S. y N. Schady (2013), "Latin America's social policy challenge: education, social insurance, redistribution", Journal of Economic Perspectives, vol. 27, № 2, Nashville, Tennessee, American Economic Association.

Lustig, N., L. F. López-Calva y E. Ortiz-Juárez (2013), "Declining inequality in Latin America in the 2000s: the cases of Argentina, Brazil, and Mexico", World Development, vol. 44, Amsterdam, Elsevier.

Mancha, T., N. Perticarari y F. Buchieri (2011), "Impactos de la crisis financiera en América Latina: principales consideraciones para una discusión”, Documento de Trabajo, № 02/2011, Madrid, Universidad de Alcalá.

Martín, F. (2008), "Evolución de las disparidades espaciales en América Latina. 1950-2008”, Principios. Estudios de Economía Política, № 11, Madrid, Fundación Sistema.

Medina, F. y M. Galván (2014a), "Crecimiento económico, pobreza y distribución del ingreso. Fundamentos teóricos y evidencia empírica para América Latina, 1997-2007”, serie Estudios Estadísticos, N 82 (LC/L.3689), Santiago, Comisión Económica para América Latina y el Caribe (CEPAL).

(2014b), "Sensibilidad de los índices de pobreza a los cambios en el ingreso y la desigualdad. Lecciones para el diseño de políticas en América Latina, 1997-2008”, serie Estudios Estadísticos, N 87 (LC/L.3823), Santiago, Comisión Económica para América Latina y el Caribe (CEPAL).

Mercader-Prats, M. y H. Levy (2004), "The role of tax and transfers in reducing personal income inequality in Europe's regions: evidence from EUROMOD”, EUROMOD Working Paper Series, N EM9/04, Essex, Universidad de Essex.

Milanovic, B. y R. Muñoz (2008), "La desigualdad de la distribución de la renta en América Latina: situación, evolución y factores explicativos", América Latina Hoy, vol. 48.

Molina, A., I. Amate y A. Guarnido (2011), "Economic and institutional determinants in fiscal pressure: an application to the European case", Journal of Economic Issues, vol. 45, N³, Taylor \& Francis.

Molina, A., A. Guarnido e I. Amate (2013), "Los efectos redistributivos del gasto público en la Unión Europea", eXtoikos, N 9, Instituto Econospérides.

Moller, S., A. Alderson y F. Nielsen (2009), "Changing patterns of income inequality in U.S. counties, 1970-2000", American Journal of Sociology, vol. 114, N4, Chicago, The University of Chicago Press.

Morgan, J. y N. Kelly (2013), "Market inequality and redistribution in Latin America and the Caribbean", The Journal of Politics, vol. 75, N³, Chicago, The University of Chicago Press.

Muinelo, L. y O. Roca (2013), "Joint determinants of fiscal policy, income inequality and economic growth", Economic Modelling, vol. 30, Amsterdam, Elsevier.

Nygard, F. y A. Sandström (1981), Measuring Income Inequality, Estocolmo, Almqvist \& Wiksell.

Pena, J. B. y otros (1996), Distribución personal de la renta en España, Madrid, Ediciones Pirámide.

Perticara, M. (2012), "Introducción", Pobreza, desigualdad de oportunidades y políticas públicas en América Latina, Río de Janeiro, Fundación Konrad Adenauer. 
Peters, D. J. (2013), "American income inequality across economic and geographic space, 1970-2010", Social Science Research, vol. 42, № 6, Amsterdam, Elsevier.

Piketty, T. (2014), El capital en el siglo XXI, Madrid, Fondo de Cultura Económica.

Quenan, C. (2013), "América Latina frente a la crisis económica internacional: buena resistencia global y diversidad de situaciones nacionales", IdeAs, № 4, Vanves, Institut des Amériques.

Ravallion, M. y S. Chen (1997), "What can new survey data tell us about recent changes in distribution and poverty?", The World Bank Economic Review, vol. 11, № 2, Washington, D.C., Banco Mundial.

Ruiz-Castillo, J. (1987), "La medición de la pobreza y la desigualdad en España, 1980-81", Estudios Económicos, № 42, Banco de España.

SEDLAC (Base de Datos Socioeconómicos para América Latina y el Caribe) [en línea] http://sedlac.econo. unlp.edu.ar/.

Shorrocks, A. F. (1980), "The class of additively decomposable inequality measures", Econometrica, vol. 48, $N^{\circ}$ 3, Nueva York, The Econometric Society.

Theil, H. (1967), Economics and Information Theory, Amsterdam, North Holland Publishing.

Villaverde, J. (1996), "Desigualdades provinciales en España, 1955-1991", Estudios Regionales, № 45, Málaga, Universidad de Málaga.

Zubiri, I. (1985), "Una introducción al problema de la medición de la desigualdad", Hacienda Pública Española, $N^{\circ}$ 95, Madrid, Instituto de Estudios Fiscales. 